\title{
MicroRNA-188-5p inhibits the progression of breast cancer by targeting zinc finger protein 91
}

\author{
ZHIGUANG YANG, ZHAOYU LIU, ZHIHUI CHANG and GUANGXIN YAN \\ Department of Radiology, Shengjing Hospital of China Medical University, Shenyang, Liaoning 110004, P.R. China
}

Received February 15, 2020; Accepted July 16, 2020

DOI: $10.3892 /$ or.2020.7731

\begin{abstract}
Breast cancer (BC) is the most commonly diagnosed malignant cancer in women. $\mathrm{BC}$ is the main cause of cancer-related death in women and seriously threatens the life and health of women worldwide. MicroRNAs (miRNAs/miRs) have been reported to regulate the development and progression of different types of cancer. However, the regulatory functions of miR-188-5p in BC have not been thoroughly demonstrated. In this present research, we identified that miR-188-5p was downregulated in $\mathrm{BC}$ tissues and several BC cell lines. Downregulation of miR-188-5p was significantly associated with advanced TNM stage. Moreover, we identified that miR-188-5p mimics significantly inhibited proliferation using CCK-8 assay, colony formation and xenograft animal model, suppressed invasion and migration detected by Transwell invasion assay, and increased the cellular apoptosis of BC cells as determined by cell apoptosis assay. Moreover miR-188-5p mimics also reduced the expression of NF- $\kappa \mathrm{B}$ p65(Rel). To further investigate its regulatory mechanism, transcription factor zinc finger protein 91 (ZFP91) was predicted as the targeted protein of miR-188-5p by bioinformatic method. We confirmed their specific binding by dual luciferase (DLR) assay. We demonstrated that the overexpression of miR-188-5p significantly inhibited the expression of ZFP91 in BC cell lines and reduced the expression of $\mathrm{NF}-\kappa \mathrm{B}$ p65(Rel). An inverse correlation was found between the expression of miR-188-5p and ZFP91 in BC tissues. Importantly, we demonstrated that the restoration of ZFP91 was able to block the effect of miR-188-5p on the progression of MDA-MB-231 cells. Therefore, our study showed that miR-188-5p may be one of the important indicators and could inhibit the progression of human BC via targeting the ZFP91/NF- $\mathrm{BB}$ p65(Rel) signaling pathway, suggesting that miR-188-5p may be a promising future target for BC treatment.
\end{abstract}

Correspondence to: Dr Zhaoyu Liu, Department of Radiology, Shengjing Hospital of China Medical University, 36 Sanhao Street, Shenyang, Liaoning 110004, P.R. China

E-mail: liuzy1226@126.com

Key words: breast cancer, miR-188-5p, zinc finger protein 91, ZFP91, proliferation, apoptosis

\section{Introduction}

Breast cancer (BC) is one of the most commonly diagnosed malignancy in the world. The mortality rate for $\mathrm{BC}$ ranks first among all female malignant tumors (1). Globally, the number of newly diagnosed BC cases reached approximately 2.1 million in 2018, accounting for almost $25 \%$ of cancer cases among women (2). BC exhibits a complex pathogenesis and is a clinically heterogeneous disease with a wide range of clinical behaviors and treatment responses (1). Although many dysregulated molecular pathways have been discovered in $\mathrm{BC}$, the development of effective therapeutic methods has been limited (3). It is urgent to discover novel molecules to suppress $\mathrm{BC}$ proliferation, induce apoptosis and inhibit invasion, and provide potential therapeutic strategies to improve the survival and quality of life of BC patients (4).

MicroRNAs (miRNAs/miRs) are a class of endogenous non-coding RNAs of approximately 19-24 nucleotides in length, which are generally located in unstable regions of the human genome and are usually dysregulated in malignant tumors to regulate gene functions (5). miRNAs regulate target genes through binding to the 3'untranslated regions (3'UTRs) of the target mRNAs, subsequently inhibiting gene expression (6). Through regulation of the targeted proteins, miRNAs play an important role in many tumor cellular processes such as proliferation, cell cycle, apoptosis, invasion and metastasis, and participate in almost all signaling pathways. miR-188-5p has been reported to be an inhibitor of tumor growth and metastasis in prostate cancer (7) and hepatocellular carcinoma (8). However, to the best of our knowledge, the functions of miR-188-5p in $\mathrm{BC}$ remain elusive.

In the present study, we detected the expression of miR-188-5p in tumor tissues of BC patient tissues and several $\mathrm{BC}$ cell lines. Furthermore, we investigated its regulatory role in $\mathrm{BC}$ proliferation, apoptosis and invasion. We also predicted and confirmed the targeted protein of miR-188-5p, transcription factor zinc finger protein 91 (ZFP91), elucidating the regulatory mechanisms of miR-188-5p in BC.

\section{Materials and methods}

Patients and tissues. One hundred paired BC tissue specimens including malignant and normal tissues used in this study were obtained from Shengjing Hospital of China Medical University (Shenyang, Liaoning, China) during the period 
from January 2017 to December 2018 with the informed consent of patients. The age range of the patients was from 31 to 75 years, with a mean age of 48.28 years. All experiments were approved by the Ethics Committee of Shengjing Hospital of China Medical University (no. 2016PS18J). The samples were snap-frozen in liquid nitrogen and stored at $-80^{\circ} \mathrm{C}$. TNM staging system was performed for tumor grading of $\mathrm{BC}$ and for evaluating and staging of patients, respectively, which was carried out according to the 7th edition of the American Joint Committee on Cancer (AJCC) TNM classification system (9).

Cell cultivation. The BC cell lines MDA-MB-231 (ATCC, CRM-HTB-26), BT-549 (ATCC, HTB-122), and MCF-7 (ATCC, CRL-3435) were cultured in RPMI-1640 medium (Sigma-Aldrich; Merck KGaA) containing 10\% heat-inactivated fetal bovine serum (FBS) (MP Biomedicals), $1 \%$ penicillin-streptomycin (Invitrogen; Thermo Fisher Scientific, Inc.; no. 15070-063). The nonmalignant mammary epithelial cell line, MCF 10A (ATCC, CRL-10317), was cultivated in DMEM/F12 Ham's Mixture supplemented with 5\% Equine Serum (Hyclone; GE Healthcare), EGF (20 ng/ml), insulin $(10 \mu \mathrm{g} / \mathrm{ml})$, hydrocortisone $(0.5 \mathrm{mg} / \mathrm{ml})$, and cholera toxin (100 ng/ml) (all from Sigma-Aldrich; Merck KGaA). All cells were incubated at $37^{\circ} \mathrm{C}$ in a humidified $5 \% \mathrm{CO}_{2}$ atmosphere.

RNA isolation and quantitative (q)PCR. Total RNAs from tissues or cells were isolated using $\mathrm{RNX}^{\mathrm{TM}}$-Plus Reagent (Cinnagen) and cDNA was synthesized using the PrimeScript ${ }^{\mathrm{TM}}$ RT Reagent Kit (Takara) according to the manufacturer's instructions. qPCR was performed using a SYBR Premix ExTaq $^{\mathrm{TM}}$ kit (Takara), with the following primer sets on the ABI 7300 qPCR system (Applied Biosystems). $\beta$-actin was used to normalize the relative expression of the target genes. miRNAs were detected through a miScript II RT kit (Qiagen) in a fluorescence thermal cycler (Bio-Rad Laboratories, Inc.). The primers for miR-188-5p and the reference gene U6 were purchased from Novland Biopharm. The thermocycling condition were $95^{\circ} \mathrm{C}$ for $10 \mathrm{~min}$, followed by 40 cycles of $95^{\circ} \mathrm{C}$ for $15 \mathrm{sec}$ and $60^{\circ} \mathrm{C}$ for $1 \mathrm{~min}$, followed by a hold at $4^{\circ} \mathrm{C}$. The relative expression ratio of miR-188-5p was quantified using the $2^{-\Delta \Delta \mathrm{Cq}}$ method (10). The relative expression of miR-188-5p was normalized to U6. The primer sequences are listed in Table I.

Plasmid preparation. The coding region of human ZFP91 was amplified from human breast cancer cell MDA-MB-231 cDNA library by PCR. Then we cloned the prepared ZFP91 fragment into pCMV-Tag2B (Stratagene) to obtain pCMV-Tag2B-ZFP91. The primer sequences are listed in Table I.

Cell transfection. The miR-188-5p mimics and miR-NC were purchased from Thermo Fisher Scientific, Inc. Firstly, Lipofectamine 2000 transfection reagent (Thermo Fisher Scientific, Inc.) was used to transfect miR-188-5p mimics, miR-NC into MDA-MB-231 cells in accordance with the manufacturer's instructions.

After the whole detection, the pCMV-Tag2B vector (PC) was transfected into MDA-MB-231 cells with miR-NC (NC+PC) or miR-188-5p mimics (PC+miR-188-5p); pCMV-Tag2B-ZFP91 was transfected into MDA-MB-231 cells with miR-188-5p mimics (miR-188-5p+ZFP91). As the cells were grown to $90 \%$, miR mimics or vector was transfected into cells according to the manufacturer's instructions. Then cells were cultivated for up to $72 \mathrm{~h}$. Finally, the total RNA and protein were extracted and properly stored for further research.

Cell proliferation assay. Cell Counting Kit-8 (CCK-8 Kit, Dojindo) was performed to detect cell proliferation based on the reduction of WST-8 to WST-8 formazan. Briefly, the MDA-MB-231 cells were seeded in a 96-well plate at a density of $5 \times 10^{3}$ cells/well. On the day of the experiment, the cells were transfected with empty vector and miR-188-5p. CCK-8 reagent was added into the culture medium at the indicated time and incubated for $60 \mathrm{~min}$; the absorbance at $450 \mathrm{~nm}$ was measured by a microplate reader.

Colony formation assay. MDA-MB-231 cells from the different treated groups were seeded in a $60-\mathrm{mm}$ dish containing 200 cells, followed by a 14-day cultivation at $37^{\circ} \mathrm{C}$ with $5 \% \mathrm{CO}_{2}$. The supernatant was discarded and cells were washed twice with PBS. The colonies were fixed in $4 \%$ paraformaldehyde for $15 \mathrm{~min}$ and then stained with Giemsa staining solution (Solarbio Science \& Technology, Co., Ltd., Beijing, China) for $20 \mathrm{~min}$. Colonies were counted and images were captured under an inverted microscope (Nikon, Tokyo, Japan). This assay was repeated 3 times.

Cell apoptosis assay. MDA-MB-231 cells were stained by Annexin V-Alexa Fluor-488/propidium iodide (PI) staining to identify the apoptotic MDA-MB-231 cells. After transfection with miR-188-5p for $48 \mathrm{~h}$, MDA-MB-231 cells were stained with Annexin V-Alexa Fluor-488 for $15 \mathrm{~min}$ on ice, followed by the addition of PI solution for the secondary staining process. All experimental procedures were strictly protected from lights. The data were calculated by FlowJo software v.8.7 (Tree Star) after FACs Calibur (BD Biosciences) analysis.

Cell migration and invasion assays. After the counting, MDA-MB-231 cells in the different groups were inoculated equally at a density of $5 \times 10^{5}$ cells $/ \mathrm{ml}$ in the upper compartments of polycarbonate membrane filters. Cell migration and invasion assays were performed; uncoated for the migration assay and coated with Matrigel (1:8, BD Biosciences) for the invasion assay. After $24 \mathrm{~h}$, the migrated and invaded cells in the membrane were fixed with methanol, and then stained with $0.1 \%$ crystal violet for $20 \mathrm{~min}$ at room temperature. Cells were observed under a light microscope with magnification, $\mathrm{x} 100$.

Western blotting. Protein samples extracted from tissues or cultivated cells were lysed in RIPA buffer containing protease and phosphatase inhibitor cocktail and incubated at $4^{\circ} \mathrm{C}$, followed by the quantified measurement of protein using $\mathrm{BCA}$ kit (FUJIFILM Wako Pure Chemical Corp.). After protein samples (40 $\mu \mathrm{g} /$ each sample) were loaded and separated on $10 \%$ SDS-PAGE gels for electrophoresis, the proteins were then transferred onto a polyvinylidene difluoride (PVDF) membranes (Millipore, USA). The membranes were blocked in $5 \%(\mathrm{w} / \mathrm{v})$ skim milk for $1 \mathrm{~h}$ at room temperature and incubated at $4^{\circ} \mathrm{C}$ overnight with primary antibodies anti-ZFP91 (dilution 
Table I. Primer information.

\begin{tabular}{ll}
\hline Gene name & \multicolumn{1}{c}{ Bi-directional primer sequence } \\
\hline miR-188-5p & F: 5'-CCCTCTCTCACATCCCTTGCAT-3' \\
& R: 5'-ATCCTGCAAACCCTGCATGTG-3' \\
ZFP91 & F: 5'-TGAGACCTACAAACCCCACTT-3' \\
& R: 5'-CCTTTTGGGTAAACGTGGACTTT-3' \\
Homo- $\beta$-actin & F: 5'-TTCCTCCGCAAGGATGACACGC-3' \\
& R: 5'-CCTTTTGGGTAAACGTGGACTTT-3' \\
U6 snRNA & F: 5'-CGGGTTTGTTTTGCATTTCT-3' \\
& F: 5'-AGTCCCAG CATGAACAGCTT-3'
\end{tabular}

ZFP91, Zinc finger protein 91 homolog; F, forward; R, reverse.

1:1,000, Abcam, ab30970) and anti-vimentin (dilution 1:1,000, Cell Signaling Technology, Inc., \#3932), anti-E-cadherin (dilution 1:1,000, Cell Signaling Technology, Inc., \#3195), N-cadherin (dilution 1:1,000, Cell Signaling Technology, Inc., \#13116), matrix metalloproteinase (MMP)-2 (dilution 1:1,000, Cell Signaling Technology, Inc., \#40994), MMP-9 (dilution 1:1,000, Cell Signaling Technology, Inc., \#13667), NF- $\kappa$ B p65 (dilution 1:1,000, Cell Signaling Technology, Inc., \#8242), RelB (dilution 1:1,000, Cell Signaling Technology, Inc, \#4954) and GAPDH (dilution 1:5,000, Cell Signaling Technology, Inc, \#5174) as internal control. On the following day, all membranes were incubated with anti-rabbit IgG HRP-labeled secondary antibodies (dilution 1:2,000, Cell Signaling Technology, Inc., \#7074). Finally, the signals were detected and analyzed with the application of Luminata Forte Western HRP Substrate (Millipore) in the Bio-Rad ChemiDox XRS+ imaging system (Bio-Rad Laboratories).

Luciferase reporter assay. To further investigate the specific correlation between miR-188-5p and ZFP91, Targetscan (www.targetscan.org/mamm_31/) and miRanda (www. microrna.org/microrna/home.do) were performed. The ZFP91 was selected to be the predicted targeting of miR-188-5p. The fragments of the 3'UTR of ZFP91 containing miR-188-5p binding sites and its mutants were amplified by PCR, and then the PCR products were inserted into pmirGLO dual-luciferase miRNA target expression vector (Promega Corp.). The reporter and control vector were transfected into $293 \mathrm{~T}$ cells using Lipofectamine 3000 (Thermo Fisher Scientific, Inc.). After cultivation for $48 \mathrm{~h}$, the relative luciferase activity was examined by the Dual-Luciferase Reporter Assay Kit (Thermo Fisher Scientific, Inc.) according to the manufacturer's instructions.

Preparation of tumor xenograft animal model and treatment with miR-188-5p mimics. Thirty-six nude mice, female, weighing $20 \pm 5 \mathrm{~g}$, were purchased from Huafukang Biotech (Beijing, China). The experiments were performed in the animal facility at the Department of Laboratory Animal Science of China Medical University and approved by the Animal Ethics Committee of Shengjing Hospital (approval no. 2019218). Nude mice were randomly divided into a control group $(n=12)$, miR-188-5p group $(n=12)$, and NC group $(n=12)$. A density of $5 \times 10^{6}$ cells in logarithmic phase were transfected with 1X PBS (control group), NC or miR-188-5p. Then the different groups of cells were resuspended in 1X PBS and injected into the nude mice, respectively. Then tumor size was measured every 3 days using a slide caliper and the tumor volume (V) was calculated using the formula: $V=$ length $x$ width $^{2} / 2$. After 21 days, the mice were euthanasia by cervical dislocation and the tumors were excised, imaged, weighed and stored properly for further investigations.

Statistical analysis. GraphPad Prism 8 (GraphPad Software, Inc.) was used to perform statistical analysis. The results are represented as mean \pm SD of at least 3 independent experiments. The comparisons between groups were evaluated by Student' t-test. One-way ANOVA followed by Tukey test was used to evaluate the differences for multiple comparisons. The statistical significance of correlations between miR-188-5p and ZFP91 expression in BC tissue were analyzed by Pearson's correlation coefficient. $\mathrm{P}<0.05$ was considered to indicate a statistically significant difference.

\section{Results}

miR-188-5p is significantly decreased in BC tissue and cell lines. Firstly, we analyzed the expression level of miR-188-5p in 100 cases of BC tissues and adjacent counterparts by RT-qPCR. The results showed that the level of miR-188-5p in $\mathrm{BC}$ tissues was significantly lower than that in the normal adjacent counterparts (Fig. $1 \mathrm{~A}, \mathrm{P}<0.05$ ). We also found that miR-188-5p was correlated with BC TNM stage (Fig. 1B, $\mathrm{P}<0.05)$. The expression level of miR-188-5p in advanced $\mathrm{BC}$ tumors was lower than that in early stage tumors, suggesting that miR-188-5p is inversely correlated with the malignancy of BC.

We also compared the expression level of miR-188-5p in the non-malignant mammary epithelial cell line MCF-10A and BC cell lines MDA-MB-231, BT-549 and MCF-7. Our data showed that the levels of miR-188-5p in the MDA-MB-231, BT-549 and MCF-7 cells were lower than that in the MCF-10A cells (Fig. $1 \mathrm{C}, \mathrm{P}<0.05$ ). Meanwhile, the lowest miR-188-5p expression was detected in MDA-MB-231, therefore, MDA-MB-231 cells were selected for further experiments.

miR-188-5p inhibits proliferation, induces cell apoptosis and suppresses migration and invasion of BC cells. As the expression of miR-188-5p in both BC cell lines and tumor tissues of $\mathrm{BC}$ patients were clearly downregulated, we sought to investigate the effects of miR-188-5p on BC development by using both in vitro $\mathrm{BC}$ cell line cultivation and in vivo mouse tumor xenografts. As shown in Fig. 2A, transfection of MDA-MB-231 cells with miR-188-5p mimics significantly elevated the expression level of miR-188-5p when compared to the control and miR-NC groups $(\mathrm{P}<0.05)$. Importantly, the increased level of miR-188-5p in MDA-MB-231 cells significantly inhibited the cell proliferation when compared to the control and miR-NC groups (Fig. $2 \mathrm{~B}$ and $\mathrm{C}, \mathrm{P}<0.05$ ). It was also observed that the apoptotic MDA-MB-231 cell numbers were significantly increased by the upregulation of miR-188-5p when compared to the control and miR-NC groups (Fig. 2D, 

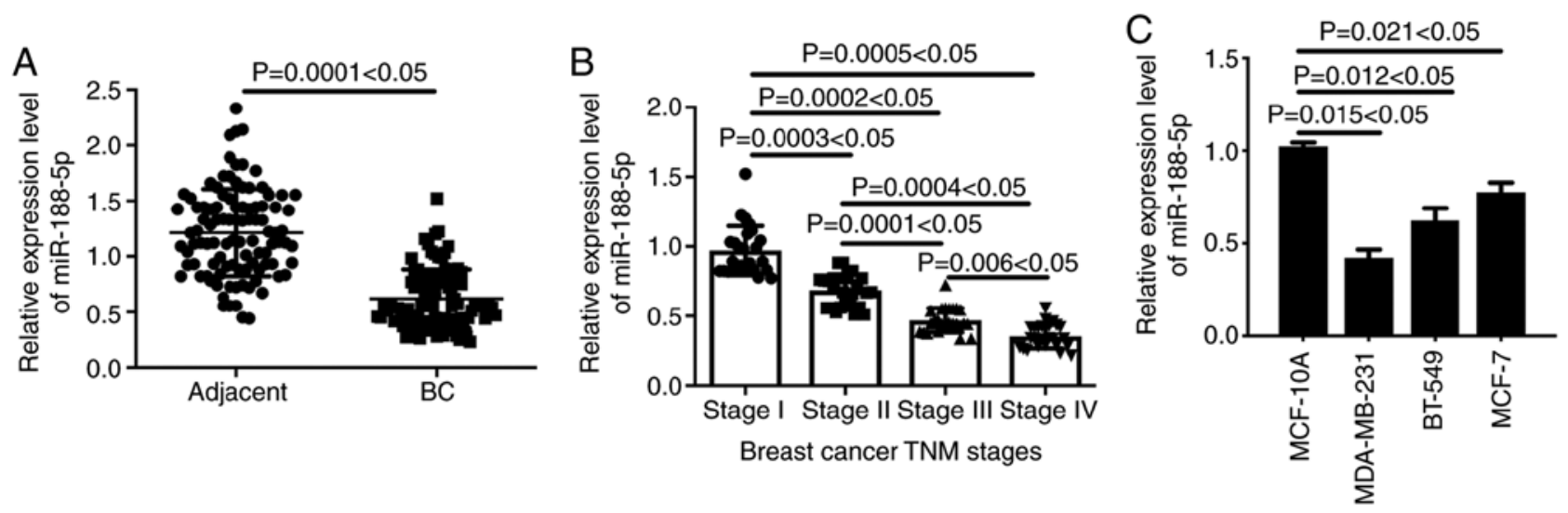

Figure 1. miR-188-5p is downregulated in BC tissue and BC cell lines. (A) RT-qPCR results showed that the level of miR-188-5p in BC tissues was downregulated compared with counterpart BC tissues. The comparisons between groups were evaluated by Student's t-test. (B) Relative expression level of miR-188-5p in patients at different clinical stages. (C) Relative expression level of miR-188-5p in BC cell lines MDA-MB-231, BT-549 and MCF-7 relative to the normal human breast epithelial cell line MCF-10A. One-way ANOVA followed by Tukey's test was used to evaluate the difference for multiple comparisons in $\mathrm{B}$ and C. All data are presented as means $\pm \mathrm{SD}(\mathrm{n}=3)$. BC, breast cancer.

$\mathrm{P}<0.05)$. Importantly, miR-188-5p mimics significantly inhibited the invasion and migration abilities of the MDA-MB-23 cells under Transwell assay detection when compared to the control and miR-NC groups (Fig. 2E, $\mathrm{P}<0.05$ ). Moreover, miR-188-5p mimics significantly enhanced the expression of vimentin and $\mathrm{N}$-cadherin and reduced the level of E-cadherin when compared to the control and miR-NC groups (Fig. 2F, $\mathrm{P}<0.05$ ). The matrix metalloproteinases, MMP2 and MMP9 (MMP2/9), possess the ability to hydrolyze components of the basement membrane and stimulate tumor growth, metastasis and epithelial-mesenchymal transition (EMT) (11). miR-188-5p mimics were demonstrated to significantly inhibit the expression of MMP2 and MMP9 (Fig. 2F, P<0.05). These data provide robust evidence that miR-188-5p inhibits the tumor proliferation, induces apoptosis, reduces tumor invasion and migration and inhibits EMT of BC, which may be through the regulation of MMP2/9 expression.

ZFP91 is the downstream target of miR-188-5p. To further investigate the specific correlation between miR-188-5p and ZFP91, Targetscan (www.targetscan.org/mamm_31/) and miRanda (www.microrna.org/microrna/home.do) were performed. The results predicted that miR-188-5p possesses the binding sites of ZFP91 (Fig. 3A). Hence, we sought to discover the regulatory mechanisms of miR-188-5p on BC development through targeting on ZFP91. As hypothesized, the upregulation of miR-188-5p in MDA-MB-231 cells decreased ZFP91 mRNA and protein levels when compared to the miR-NC and control groups (Fig. 3B, $\mathrm{P}<0.05$ ). Moreover, the luciferase assay confirmed that miR-188-5p specifically binds to the 3'UTR of ZFP91 (Fig. 3C, P<0.05). It was also discovered that the injection of MDA-MB-231 transfected with miR-188-5p mimics in tumor xenograft mice inhibited ZFP91 expression (Fig. 6B, P<0.05). These results suggested that ZFP91 is the downstream target gene of miR-188-5p in BC.

miR-188-5p regulates $B C$ cell progression through targeting ZFP91. To further investigate the biological functions of miR-188-5p in BC development, we established a ZFP91-overexpressing MDA-MB-231 cell line. The expression of ZFP91 was confirmed by RT-qPCR (Fig. S1, P<0.05). With this system, inhibition of ZFP91 by miR-188-5p mimics was reversed (Fig. 4A, $\mathrm{P}<0.05$ ). Then it was found that the co-transfection of MDA-MB-231 cells, miR-188-5p+ZFP91 group, significantly enhanced the cell proliferation compared to that in PC+miR-188-5p group (Fig. 4B and C, P<0.05), significantly suppressed cell apoptosis (Fig. 4D, $\mathrm{P}<0.05$ ) and significantly promoted invasion, migration (Fig. $4 \mathrm{E}, \mathrm{P}<0.05$ ) and EMT (Fig. 4F, P<0.05), in contrast with the mono-transfection of miR-188-5p mimics in MDA-MB-231 cells. Moreover, the regulatory role of miR-188-5p on MMP2 and MMP9 was also reversed by overexpression of ZFP91.

miR-188-5p and ZFP91 are correlated in tumor tissues of $B C$ patients. Furthermore, we examined the levels of ZFP91 in the tumor tissues and adjacent normal tissues of BC patients. The aberrantly high level of ZFP91 was observed in the tumor tissues of the BC patients. (Fig. 5A, P<0.05). Spearman's correlation analysis showed a significantly inverse correlation between miR-188-5p and ZFP91 in the BC patient tissues (Fig. 5B, $\mathrm{P}<0.05$ ). Taken together, these results further confirmed that the proliferation and apoptosis of $\mathrm{BC}$ is regulated by miR-188-5p/ZFP91.

miR-188-5p inhibits the proliferation of MDA-MB-231 cells and reduces the expression of ZPF91 in a BC xenograft mouse model. Moreover, to evaluate the regulatory role of miR-188-5p in a BC xenograft mouse model, we injected the MDA-MB-231 cells transfected with miR-188-5p mimics or miR-NC into nude mice. The results showed that miR-188-5p mimics inhibited the tumor volume and weight compared to the miR-NC group (Fig. 6A, $\mathrm{P}<0.05$ ). Protein expression and the mRNA level of ZPF91 were also suppressed by miR-188-5p mimics in the tumor tissues of the xenograft mouse model when compared with the miR-NC and control groups (Fig. 6B, $\mathrm{P}<0.05$ ).

miR-188-5p/ZFP91 axis regulates $N F-k B / P 65$ and RelB expression. Numerous studies have reported that zinc finger protein 91 (ZFP91) promotes proliferation and tumorigenesis 

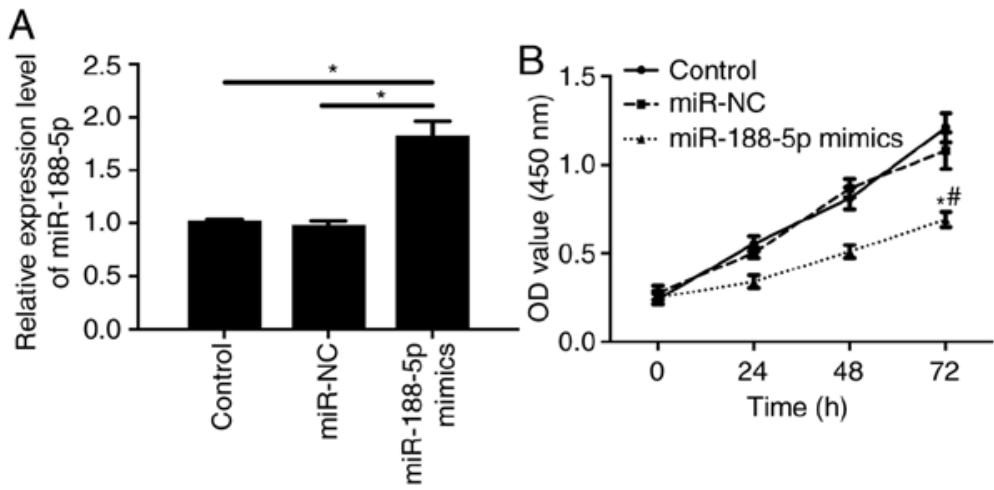
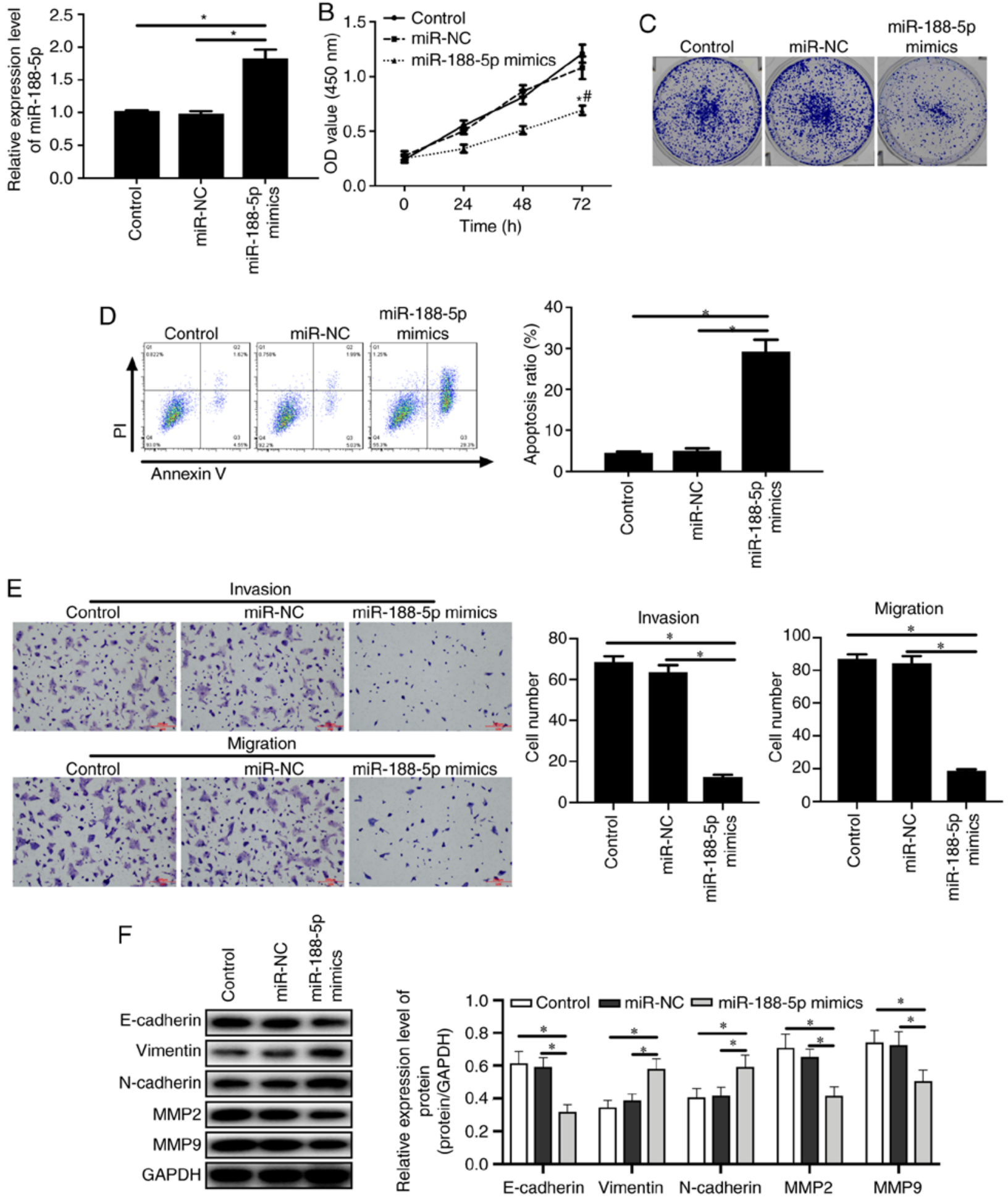

Figure 2. Effect of miR-188-5p on BC cell line MDA-MB-231. MDA-MB-231 cells were transfected with miR-188-5p mimics and miR-NC. (A) The mRNA levels of miR-188-5p in MDA-MB-231 cells were determined by RT-qPCR. "P<0.05. The proliferation of MDA-MB-231 cells was examined by (B) CCK-8 Kit post transfection and (C) colony formation. ${ }^{*} \mathrm{P}<0.05$, compared to control group; ${ }^{\prime \prime} \mathrm{P}<0.05$, compared to miR-NC group. (D) The apoptotic MDA-MB-231 cells were analyzed using Annexin V/PI staining and FACs. " $\mathrm{P}<0.05$. (E) The invasion and migration capability of MDA-MB-231 cells were detected by Transwell assay. "P<0.05. (F) Expression of E-cadherin, N-cadherin, vimentin, MMP2, MMP9 and NF-kB p65(Rel) were detected by western blotting. The data are represented as the mean $\pm \mathrm{SD}(\mathrm{n}=3)$. ${ }^{*} \mathrm{P}<0.05$. One-way ANOVA followed by Tukey's test was used to evaluate the difference for multiple comparisons. $\mathrm{BC}$, breast cancer; MMP, matrix metalloproteinase; $\mathrm{NC}$, negative control.

of different cancer types via regulation of the NF- $\kappa \mathrm{B}$ p65 pathway (12-14). Therefore, to further investigate the regulatory mechanism of the miR-188-5p/ZFP91 axis, we detected the expression of NF- $\mathrm{kBp} 65$ and RelB in BC cells. The results showed that miR-188-5p mimics significantly reduced the expression of NF- $\kappa$ Bp65 and RelB together (Fig. S2A, $\mathrm{P}<0.05)$. Moreover, the co-transfection of miR-188-5p mimics and ZFP91 also upregulated the expression levels of 

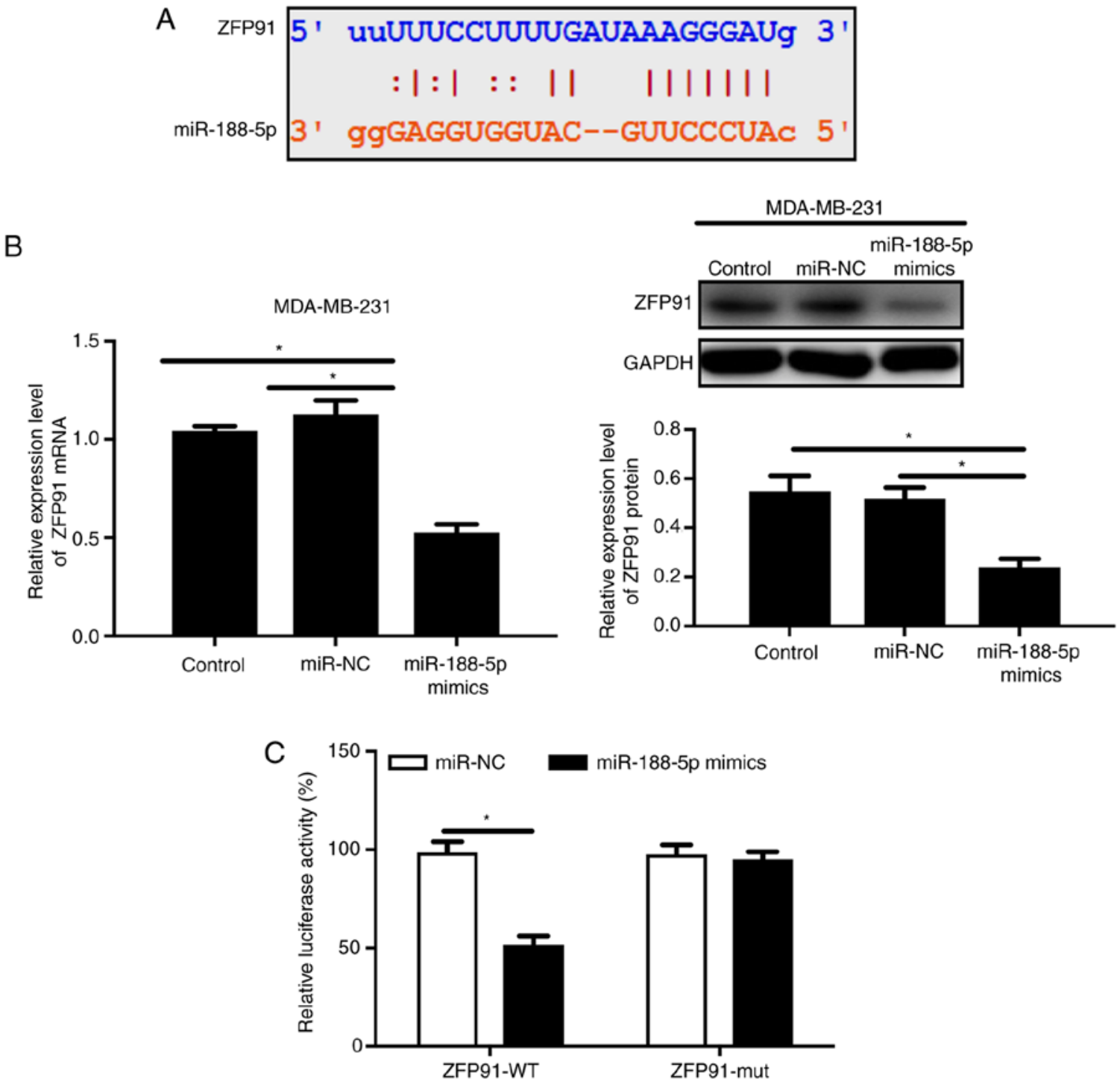

Figure 3. ZFP91 is a target of miR-188-5p. (A) ZFP91 provides binding sites with miR-188-5p. (B) RT-qPCR and western blotting were used for analysis of transcription and translation of ZFP91 in vitro and in vivo. ${ }^{~} \mathrm{P}<0.05$. (C) The binding between miR-188-5p and ZFP91 was confirmed by luciferase assay. ${ }^{*} \mathrm{P}<0.05$. Relative gene expression was normalized by GAPDH expression. The data are represented as the mean $\pm \mathrm{SD}$ ( $\mathrm{n}=3$ ). One-way ANOVA followed by Tukey's test was used to evaluate the difference for multiple comparisons in B. The comparisons between two groups were evaluated by t-test in C. ZFP91, zinc finger protein 91 .

$\mathrm{NF}-\kappa \mathrm{Bp} 65$ and RelB compared to the mono-transfection of miR-188-5p mimics (Fig. S2B, P<0.05). In summary, these results illustrated that the $\mathrm{miR}-188-5 \mathrm{p} / \mathrm{ZFP} 91$ axis regulates the progression of $\mathrm{BC}$ via the non-canonical $\mathrm{NF}-\kappa \mathrm{B}$ signaling pathway.

\section{Discussion}

Breast cancer (BC) is one of the most common types of tumors diagnosed in women worldwide (1). BC is the second leading cause of cancer-related mortality worldwide (1) (15). In 2015, more than 3,00,000 women were diagnosed with BC in China, and almost 17 percent of all newly diagnosed cancer cases were in women (1). However, the molecular mechanisms of BC still await elucidation, and effective molecular targets for the diagnosis and treatment of BC are urgently required (16). Recently, research has reported that miRNAs are small non-coding RNA molecules which regulate target protein expression to play critical roles as tumor-promotors or suppressors (17). Several studies have demonstrated that miR-188-5p promotes cell proliferation, migration and metastasis in gastric cancer $(18,19)$ and hepatocellular carcinoma (8). Moreover, Iwakawa et al detected higher expression of miR-188-5p in stage III breast cancer and TNBC (20). Wang et al reported that circulating miR-188-5p was upregulated in BC patients and associated with TNM of BC; interestingly, miR-188-5p was downregulated in BC MDA-MB-231 and MCF-7 cells. Moreover, using gain-of- and loss-of-function analyses of miR-188-5p in breast cancer cells the authors demonstrated that miR-188-5p inhibited the proliferation and invasion of BC MDA-MB-231 cells via targeting IL6ST (21). However, we demonstrated that the expression of miR-188-5p was drastically downregulated in $\mathrm{BC}$ tissue specimens, which was also decreased in BC cell lines, MDA-MB-231, BT549 and MCF-7, compared to normal breast epithelial cell line MCF-10A. Moreover, the downregulation of miR-188-5p was significantly 
A

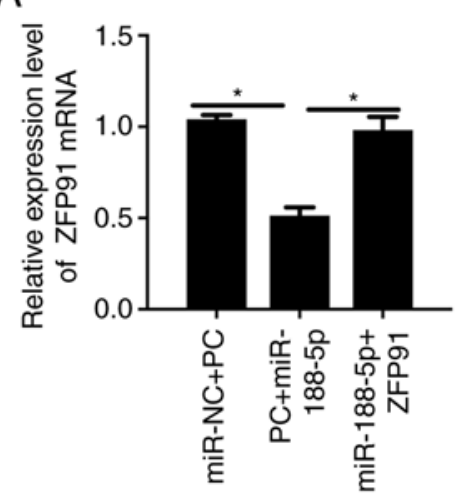

B

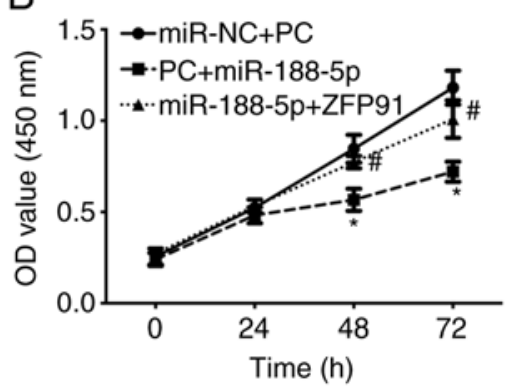

C

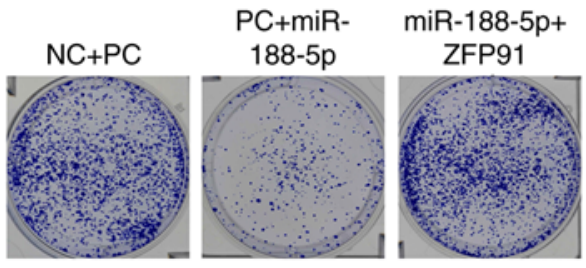

D

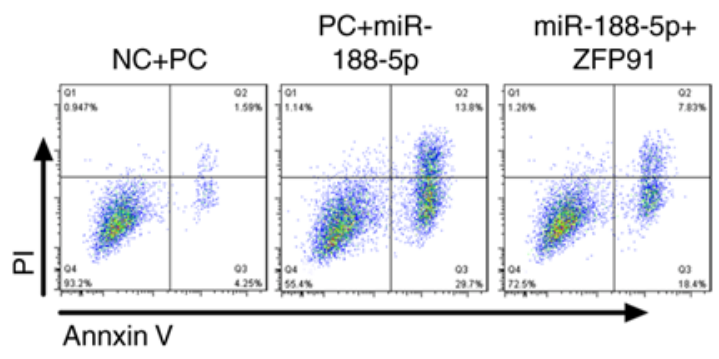

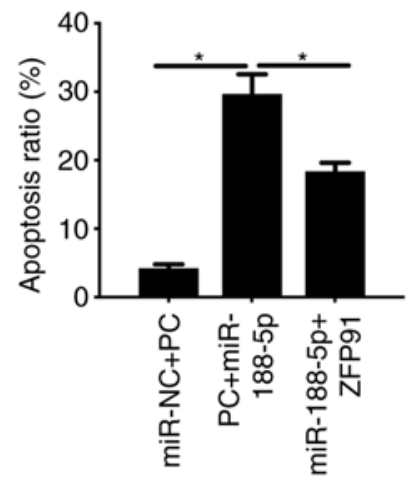

$\mathrm{E}$
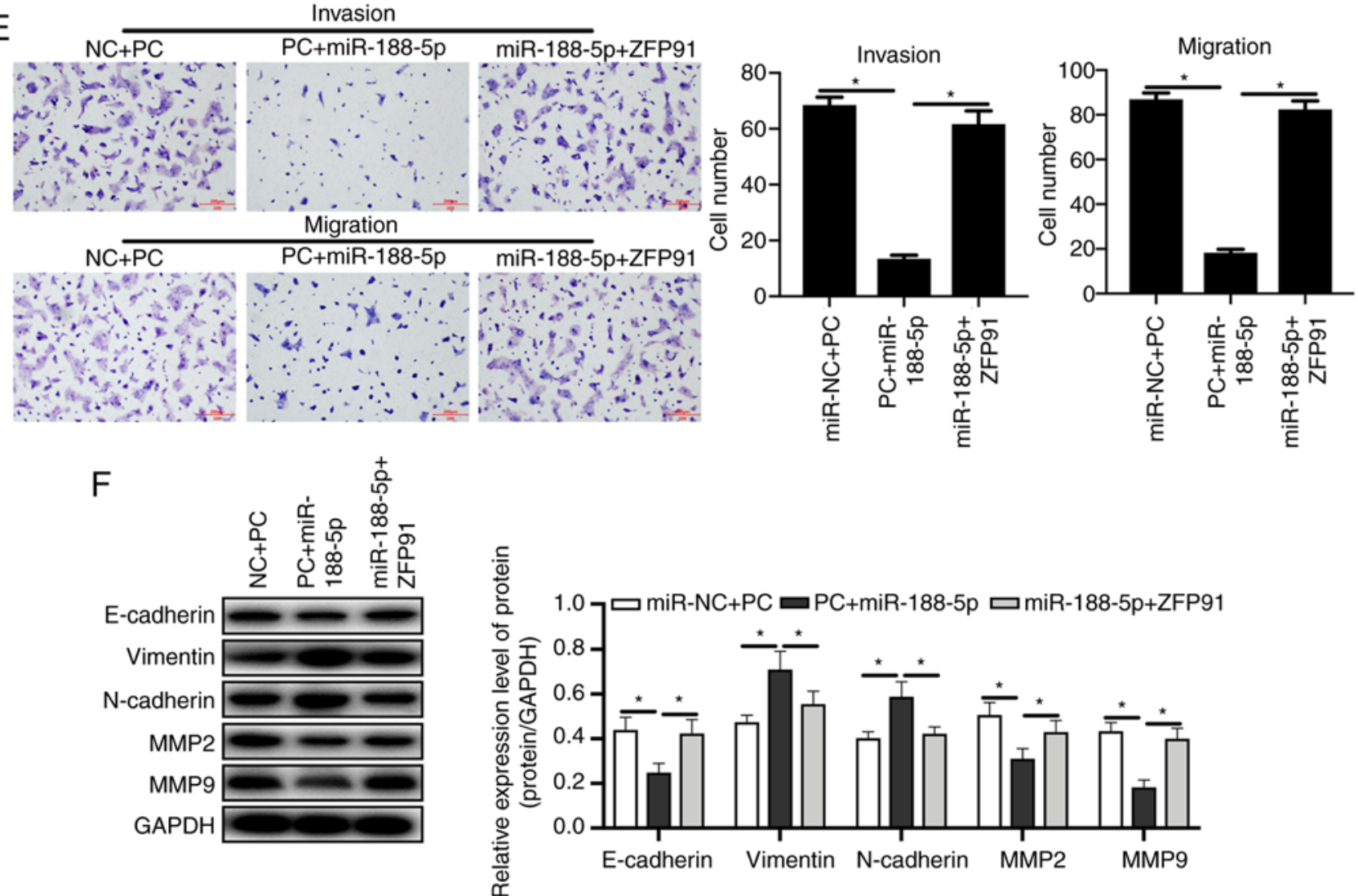

Figure 4. MDA-MB-231 cell proliferation and apoptosis are regulated by miR-188-5p/ZFP91. pCMV-Tag2B vector (PC) was transfected into BC MDA-MB-231 cells with miR-NC (NC+PC) or miR-188-5p mimics (PC+miR-188-5p); pCMV-Tag2B-ZFP91 was transfected into MDA-MB-231 cells with miR-188-5p mimics (miR-188-5p+ZFP91). (A) The mRNA levels of miR-188-5p in the co-transfected overexpressing ZFP91/MDA-MB-231 cells were quantified by RT-qPCR. "P $<0.05$. Cell proliferation was measured by (B) the CCK- 8 Kit and (C) colony formation assay. ${ }^{*} \mathrm{P}<0.05$, compared to control group; ${ }^{*} \mathrm{P}<0.05$, compared to miR-NC group. (D) Cell apoptosis was detected by Annexin V/PI staining and FACs. ${ }^{*} \mathrm{P}<0.05$. (E) The invasion and migration capability of MDA-MB-231 cells were detected by Transwell assay. "P<0.05. (F) Expression of E-cadherin, N-cadherin, Vimentin, MMP2, MMP9 and NF- $k B$ p65(Rel) were detected by western blotting. Relative genes expression was normalized by GAPDH expression. The data are represented as the mean $\pm \mathrm{SD}(\mathrm{n}=3)$. $\mathrm{P}<0.05$. One-way ANOVA followed by Tukey's test was used to evaluate the difference for multiple comparisons. BC, breast cancer; ZFP91, zinc finger protein 91; MMP, matrix metalloproteinase; NC, negative control. 
A

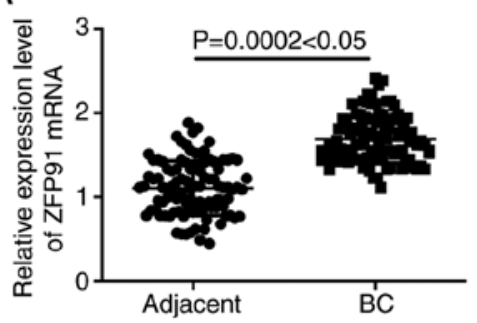

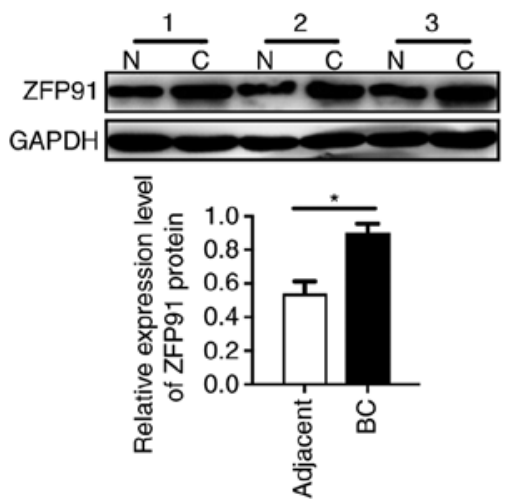

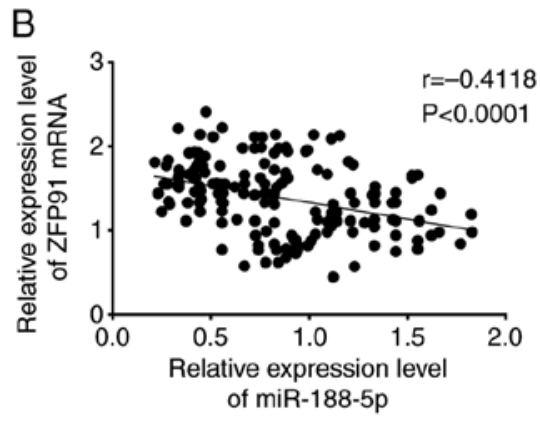

Figure 5. mRNA and protein levels of ZPF91 in BC tissues. (A) The mRNA and protein levels of ZPF91 in BC tissue and corresponding non-tumor normal tissue were quantified using RT-qPCR and western blot analysis. The comparisons between two groups were evaluated by t-test. N, non-cancer tissue; $\mathrm{C}$, cancer tissue. ${ }^{*} \mathrm{P}<0.05$. (B) Correlation between ZPF91 mRNA and miR-188-5p was analyzed using Pearson's correlation coefficient. The data are represented as the mean \pm SD $(n=100)$. BC, breast cancer; ZFP91, zinc finger protein 91 .

A
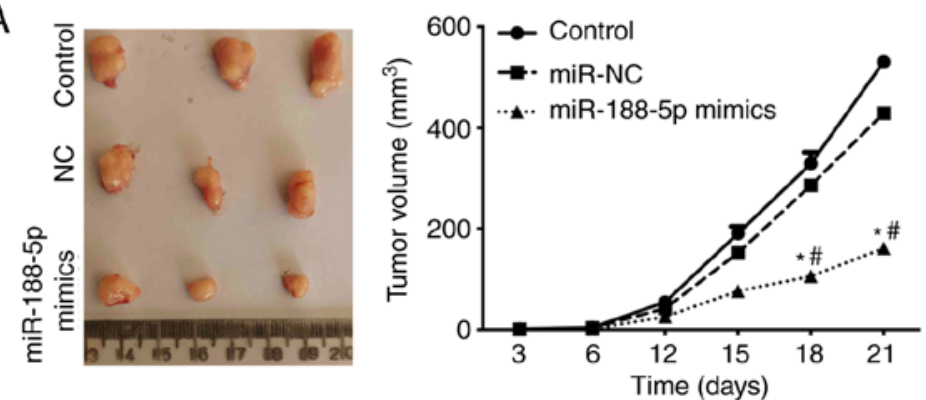

B

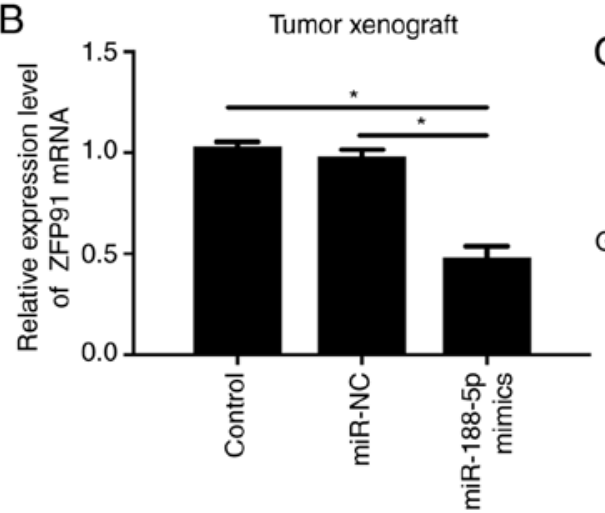

C

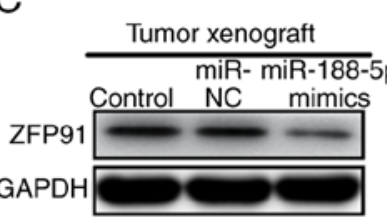

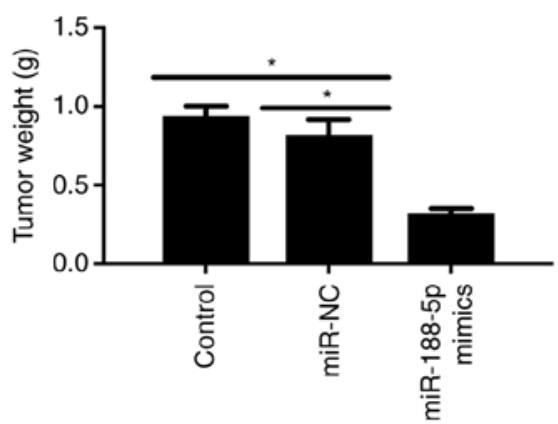

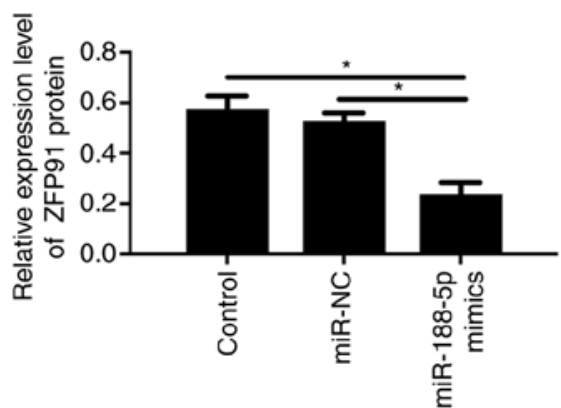

Figure 6. The regulatory role of miR-188-5p in a BC xenograft mouse model. BC MDA-MB-231 cells were transfected with miR-188-5p mimics and miR-NC, and then injected into nude mice. (A) The tumor volume and weight in the tumor xenograft mouse were compared among the miR-188-5p mimics group, miR-NC group and control group. " $\mathrm{P}<0.05$, compared to control group; ${ }^{~} \mathrm{P}<0.05$, compared to miR-NC group. (B) The protein expression and mRNA level of ZPF91 were detected by $(C)$ western blotting and RT-qPCR. The data are represented as the mean \pm SD $(n=3)$. " $P<0.05$. One-way ANOVA followed by Tukey's test was used to evaluate the difference for multiple comparisons. BC, breast cancer; ZFP91, zinc finger protein 91; NC, negative control.

associated with advanced TNM stage. However, to investigate the relationship of miR-188-5p and BC patient prognosis, we found that Kaplan-Meier analysis of miR-188-5p was limited due to the small sample size in TCGA. These results illustrated that the downregulation of miR-188-5p may be related with $\mathrm{BC}$ progression in the clinic, suggesting that miR-188-5p may be a valuable BC diagnostic indicator. Moreover, we confirmed that miR-188-5p mimics considerably inhibited the proliferation, induced the apoptosis and inhibited the invasion of BC cells, suggesting that miR-188-5p plays an inhibitory role in BC cells.
Transcription factor zinc finger protein 91 (ZFP91) was firstly identified in the mouse in 1995 (22), which was found to be overexpressed in colon (12), liver, prostate, stomach (23) and breast cancer (24). ZFP91 has a molecular mass of $63.4 \mathrm{kDa}$ with 570 amino acids, containing five zinc-finger motives, a leucine zipper, a coiled-coil structure and nuclear localization sequences. ZFP91 was confirmed to be a transcription factor located in the cellular nucleus (25). Ma et al reported that ZFP91 functions as an oncogene in cancer development by activating HIF-1 $\alpha$ transcription (12). The overexpression of ZFP91 
was also found to result in the promotion of NK- $\mathrm{B}$ signaling pathway activation through increasing $\mathrm{NK}-\kappa \mathrm{B}$ inducing kinase (NIK) (14), whose activity and overexpression are related to cancer progression in melanoma, pancreatic, breast- and lung cancer (26). The inhibition of ZFP91 was demonstrated to promote apoptosis in BC, stomach cancer cells (23), colon cancer and endometrial cancer (25). In addition, the overexpression of ZFP91 was found to increase the cancer cell growth rate and metastatic capability (23). ZFP91 was also reported to interact with cyclin-dependent kinase inhibitor 2A (CDKN2A), which is an alternative reading frame (ARF) tumor suppressor, inhibiting the induction of p53-dependent cell death (27). To illuminate the molecular mechanisms of miR-188, we predicted that IL6ST, FOXN2, ZFP91 may be the targets of miR-188-5p using Targetscan and miRanda. Furthermore, Peng et al reported that ZFP91 is the target protein of miR-188-5p in gastric cancer (28). In addition, overexpression of miR-188-5p was confirmed to inhibit the progression of breast cancer. Thus, we chose the reported oncogene ZFP91 for further investigation. In the present study, we confirmed that the 3'untranslated region (3'UTR) of ZFP91 was bound by miR-188-5p through Dual luciferase assay. Moreover, transfection of miR-188-5p mimics in MDA-MB-231 cells reduced the ZFP91 mRNA and protein levels together. miRNAs usually bind to the 3'UTRs of target mRNAs and do not reduce the level of mRNAs; however, miRNAs also were reported to decay the target mRNAs and decease mRNA level $(20,29)$. Restoration of ZFP91 largely reversed the decreased proliferation and induced apoptosis which were both regulated by miR-188-5p overexpression. Moreover, in the tumor xenograft mouse model, we observed that the expression of ZFP91 was downregulated by an increased level of miR-188-5p. Furthermore, the expression of miR-188-5p and ZFP91 were negatively correlated in BC patient tissues. Therefore, our studies confirmed that miR-188-5p can inhibit the progression of human BC via targeting ZFP91.

ZFP91 has been reported to promote proliferation in colon cancer (12), prostate cancer (13) and gastric cancer (28). Ma et al reported that ZFP91 activates NF-kappaB/p65 to promote proliferation and tumorigenesis of colon cancer (12). Paschke et al identified that ZFP91 is a noncanonical NF- $\mathrm{B}$ signaling pathway regulator with oncogenic properties in prostate cancer (13). In the present study, we also confirmed that a decrease in ZFP91 could significantly inhibit NF- $\kappa$ B/p65 and RelB expression in BC cells. Therefore miR-188-5p overexpression reduced ZFP91 via the noncanonical NF- $\kappa B$ signaling pathway to inhibit the progression of BC.

In conclusion, our data showed that miR-188-5p is downregulated in BC cell lines and tissues, and the downregulated expression of miR-188-5p is associated with the poor prognosis of patients with BC. We further investigated that overexpression of miR-188-5p could inhibit proliferation and induce the apoptosis of MDA-MB-231 cells. Furthermore, ZFP91 was predicted and confirmed as a target gene of miRNA-188-5p, and the effects of miR-188-5p on BC cells were dependent on the inhibition of ZFP91. Additionally, a decrease in ZFP91 significantly inhibited the NF- $\kappa \mathrm{B} / \mathrm{p} 65$ and RelB expression in BC cells. Moreover, the expression levels of miR-188-5p and ZFP91 were highly correlated with BC progression. Therefore, we suggest that miR-188-5p can inhibit breast cancer progression via the ZFP91/NF- $\kappa \mathrm{B} / \mathrm{p} 65$ axis and may be a potential diagnostic indicator for BC.

\section{Acknowledgements}

Not applicable.

\section{Funding}

This study was supported by the National Natural Science Foundation of China (grant nos. 81871465 and 81470086) and 345 Talent Project.

\section{Availability of data and materials}

The datasets used and analyzed during the current study are available from the corresponding author upon reasonable request.

\section{Authors' contributions}

ZY and ZL conceived and designed the study. ZY, ZC, GY performed the experiments. ZY wrote the paper. ZY, ZL, ZC and GY reviewed the results and data and edited the manuscript. All authors read and approved the manuscript and agree to be accountable for all aspects of the research in ensuring that the accuracy or integrity of any part of the work are appropriately investigated and resolved.

\section{Ethics approval and consent to participate}

Tissues used in this study were obtained from Shengjing Hospital of China Medical University (Shenyang, Liaoning, China) with the informed consent of patients, and all experiments were approved by the Ethics Committee of Shengjing Hospital of China Medical University (no. 2016PS18J).

\section{Patient consent for publication}

Not applicable

\section{Competing interests}

The authors declare that they have no competing interests.

\section{References}

1. Davidson NE, Armstrong SA, Coussens LM, Cruz-Correa MR, DeBerardinis RJ, Doroshow JH, Foti M, Hwu P, Kensler TW, Morrow M, et al: AACR cancer progress report 2016. Clin Cancer Res 22 (Suppl 19): S1-S137, 2016.

2. Ueno NT, Fernandez JRE, Cristofanilli M, Overmoyer B, Rea D, Berdichevski F, El-Shinawi M, Bellon J, Le-Petross HT, Lucci A, et al: International consensus on the clinical management of inflammatory breast cancer from the morgan welch inflammatory breast cancer research program 10th anniversary conference. J Cancer 9: 1437-1447, 2018.

3. Lim B, Woodward WA, Wang X, Reuben JM and Ueno NT: Inflammatory breast cancer biology: The tumour microenvironment is key. Nat Rev Cancer 18: 485-499, 2018.

4. Patnaik A, Rosen LS, Tolaney SM, Tolcher AW, Goldman JW, Gandhi L, Papadopoulos KP, Beeram M, Rasco DW, Hilton JF, et al: Efficacy and safety of abemaciclib, an inhibitor of CDK4 and CDK6, for patients with breast cancer, non-small cell lung cancer, and other solid tumors. Cancer Discov 6: 740-753, 2016.

5. Bartel DP: MicroRNAs: Genomics, biogenesis, mechanism, and function. Cell 116: 281-297, 2004. 
6. Filipowicz W, Bhattacharyya SN and Sonenberg N: Mechanisms of post-transcriptional regulation by microRNAs: Are the answers in sight? Nat Rev Genet 9: 102-114, 2008.

7. Zhang H, Qi S, Zhang T, Wang A, Liu R, Guo J, Wang Y and $\mathrm{Xu}$ Y: miR-188-5p inhibits tumour growth and metastasis in prostate cancer by repressing LAPTM4B expression. Oncotarget 6: 6092-6104, 2015.

8. Fang F, Chang RM, Yu L, Lei X, Xiao S, Yang H and Yang LY: MicroRNA-188-5p suppresses tumor cell proliferation and metastasis by directly targeting FGF5 in hepatocellular carcinoma J Hepatol 63: 874-885, 2015.

9. Edge SB, Byrd DR, Compton CC, Fritz AG, Greene FL and Trotti A: AJCC cancer staging manual. Vol 7. Springer, New York, NY, 2010.

10. Livak KJ and Schmittgen TD: Analysis of relative gene expression data using real-time quantitative PCR and the 2(-Delta Delta C(T)) method. Methods 25: 402-408, 2001.

11. Jacob A, Jing J, Lee J, Schedin P, Gilbert SM, Peden AA, Junutula JR and Prekeris R: Rab40b regulates MMP2 and MMP9 trafficking during invadopodia formation and breast cancer cell invasion. J Cell Sci 126: 4647-4658, 2013.

12. Ma J, Mi C, Wang KS, Lee JJ and Jin X: Zinc finger protein 91 (ZFP91) activates HIF-1 $\alpha$ via NF- $\kappa \mathrm{B} / \mathrm{p} 65$ to promote proliferation and tumorigenesis of colon cancer. Oncotarget 7: 36551, 2016.

13. Paschke L, Jopek K, Szyszka M, Tyczewska M, Ziolkowska A, Rucinski $M$ and Malendowicz LK: ZFP91: A noncanonical $\mathrm{NF}-\kappa \mathrm{B}$ signaling pathway regulator with oncogenic properties is overexpressed in prostate cancer. Biomed Res Int 2016: 6963582, 2016.

14. Jin X, Jin HR, Jung HS, Lee SJ, Lee JH and Lee JJ: An atypical E3 ligase zinc finger protein 91 stabilizes and activates NF-kappaB-inducing kinase via Lys63-linked ubiquitination. J Biol Chem 285: 30539-30547, 2010.

15. McGuire A, Brown JAL, Malone C, McLaughlin R and Kerin MJ: Effects of age on the detection and management of breast cancer. Cancers (Basel) 7: 908-929, 2015.

16. McLaughlin SA, Staley AC, Vicini F, Thiruchelvam $P$ Hutchison NA, Mendez J, MacNeill F, Rockson SG, DeSnyder SM, Klimberg S, et al: Considerations for clinicians in the diagnosis, prevention, and treatment of breast cancer-related lymphedema: Recommendations from a multidisciplinary expert ASBrS panel: Part 1: Definitions, assessments, education, and future directions. Ann Surg Oncol 24: 2818-2826, 2017.

17. Romano G, Veneziano D, Acunzo M and Croce CM: Small non-coding RNA and cancer. Carcinogenesis 38: 485-491, 2017.
18. Wang M, Qiu R, Gong Z, Zhao X, Wang T, Zhou L, Lu W, Shen B, Zhu W and Xu W: miR-188-5p emerges as an oncomiRNA to promote gastric cancer cell proliferation and migration via upregulation of SALL4. J Cell Biochem 120: 15027-15037, 2019.

19. Li Y, Yan X, Shi J, He Y, Xu J, Lin L, Chen W, Lin X and Lin X: Aberrantly expressed miR-188-5p promotes gastric cancer metastasis by activating $\mathrm{Wnt} / \beta$-catenin signaling. BMC Cancer 19: 505, 2019.

20. Iwakawa HO and Tomari Y: The functions of microRNAs: mRNA decay and translational repression. Trends Cell Biol 25 651-665, 2015.

21. Wang M, Zhang H, Yang F, Qiu R, Zhao X, Gong Z, Yu W, Zhou B, Shen B and Zhu W: miR-188-5p suppresses cellular proliferation and migration via IL6ST: A potential noninvasive diagnostic biomarker for breast cancer. J Cell Physiol 235: 4890-4901, 2020.

22. Saotome Y, Winter CG and Hirsh D: A widely expressed novel $\mathrm{C} 2 \mathrm{H} 2$ zinc-finger protein with multiple consensus phosphorylation sites is conserved in mouse and man. Gene 152: 233-238, 1995.

23. Lee JJ, Lee JH, Lee K, Hong YS and Jin XJ: Therapeutic agent for cancer, inflammation, and auto-immune disease containing inhibitor of zinc finger protein 91. US Patent 20080248024A1. Filed February 28, 2008; issued October 9, 2008.

24. Paschke L, Jopek K, Szyszka M, Tyczewska M, Malendowicz LK and Rucinski M: ZFP91 zinc finger protein expression pattern in normal tissues and cancers. Oncol Lett 17: 3599-3606, 2019.

25. Unoki M, Okutsu J, and Nakamura Y: Identification of a novel human gene, ZFP91, involved in acute myelogenous leukemia. Int J Oncol 22: 1217-1223, 2003.

26. Xiao $\mathrm{G}$ and $\mathrm{Fu} \mathrm{J}$ : NF- $\kappa \mathrm{B}$ and cancer: A paradigm of Yin-Yang. Am J Cancer Res 1: 192-221, 2011.

27. Tompkins V, Hagen J, Zediak VP and Quelle DE: Identification of novel ARF binding proteins by two-hybrid screening. Cell Cycle 5: 641-646, 2006.

28. Peng Y, Shen X, Jiang H, Chen Z, Wu J, Zhu Y, Zhou Y and Li J: miR-188-5p suppresses gastric cancer cell proliferation and invasion via targeting ZFP91. Oncol Res 27: 65-71, 2018.

29. Behm-Ansmant I, Rehwinkel J and Izaurralde E: MicroRNAs silence gene expression by repressing protein expression and/or by promoting mRNA decay. Cold Spring Harb Symp Quant Biol 71: 523-530, 2006.

(i) This work is licensed under a Creative Commons Attribution-NonCommercial-NoDerivatives 4.0 International (CC BY-NC-ND 4.0) License. 